\title{
Domain-width model for perpendicularly magnetized systems with Dzyaloshinskii-Moriya interaction
}

\author{
T. N. G. Meier, M. Kronseder, and C. H. Back* \\ Physics Department, Universität Regensburg, Universitätsstrasse 31, 93040 Regensburg, Germany \\ (Received 17 March 2017; revised manuscript received 13 September 2017; published 6 October 2017; \\ corrected 6 November 2017)
}

\begin{abstract}
The influence of the Dzyaloshinskii-Moriya interaction (DMI) on stripe domains in perpendicularly magnetized thin ferromagnetic films is theoretically and experimentally investigated. We develop a domain spacing model describing the dependence of the stripe domain width on the magnetic properties of the sample. By including the magnetostatic energy of the domain walls the model correctly describes the transition from Bloch to Néel walls with increasing DMI constant. An approach to determine the magnitude of the DMI constant by fitting the stripe domain width as a function of the effective perpendicular anisotropy of wedge-shaped samples is developed and applied to several ultrathin multilayer samples based on $\mathrm{Ni} / \mathrm{Fe} / \mathrm{Cu}(001)$. The magnitude of the DMI constant arising from $\mathrm{Fe} / \mathrm{Ni}$ and $\mathrm{Ni} / \mathrm{Fe}$ interfaces is $0.3 \pm 0.14 \mathrm{meV} / \mathrm{atom}$, indicating that the domain walls are in a pure chiral Néel state. Furthermore, phase diagrams of the skyrmionic bubble domain phase are recorded for two samples with different DMI constants, and by scaling the magnetic field a universal phase diagram for perpendicularly magnetized systems is obtained.
\end{abstract}

DOI: 10.1103/PhysRevB.96.144408

\section{INTRODUCTION}

Chiral magnetic spin textures are promising candidates for future applications in data storage and spintronics due to their topologically nontrivial spin structure, which can be unwound only by overcoming a large energy barrier [1-4]. Homochirality in ferromagnetic systems can be caused by the Dzyaloshinskii-Moriya interaction (DMI) [5-7]. On the atomic level DMI can be created, on the one hand, as a higherorder term in the direct exchange interaction with the spin-orbit coupling as a perturbation [6] (e.g., in metallic B20 compounds such as $\mathrm{MnSi}$ ); on the other hand, it can be created by an exchange interaction between two atoms of a ferromagnet mediated by a third nonmagnetic impurity or nonmagnetic overlayer consisting of atoms with large spin-orbit coupling [7] (e.g., heavy metal/ferromagnet interfaces such as Pt/Co). Due to its antisymmetric character DMI requires a broken inversion symmetry (BIS).

DMI was first investigated in noncentrosymmetric bulk crystals with complicated crystal structures, for example, the B20 structure found in some metal silicides [8-12]. However, inversion symmetry can also be broken by interfaces in thin ferromagnetic films and heterostructures. DMI, for example, leads to chiral magnetic textures in monolayers of $\mathrm{Mn}$ on $\mathrm{W}(001)$ [13,14] and $\mathrm{Fe} / \operatorname{Ir}(111)$ [15]. More recently it was shown that DMI also exists in perpendicularly magnetized ferromagnetic heterostructures, such as Pt/Co-based multilayers [4,16-18] and $\mathrm{Fe} / \mathrm{Ni} / \mathrm{Cu}(001)[19,20]$. The effect of the DMI on nucleation and motion of chiral domain walls was studied theoretically [21] and experimentally by several works on different types of perpendicularly magnetized heterostructures [16-18,22,23]. Furthermore, the existence of a phase of homochiral "skyrmionic" bubble domains in a certain outof-plane magnetic field range attracted considerable interest $[4,20,24,25]$. Several experiments on motion and manipulation

\footnotetext{
*christian.back@ur.de
}

of skyrmions or skyrmion bubbles were conducted; for example, current-driven motion of skyrmionic bubble domains was demonstrated in $\mathrm{Pt} / \mathrm{Co} / \mathrm{Ta}$ [4] and $\mathrm{Ta} / \mathrm{CoFeB} / \mathrm{TaOx}$ [26,27], and skyrmion motion was investigated in $\mathrm{MnSi}$ [2].

Furthermore, the investigation of DMI was also extended to the stripe domain phase at zero field in these systems [24,25,28]. However, most domain models, except for [28], treat only the case of strong DMI for Co/Pt-based multilayer stacks, which forces the domain walls into the pure Néel wall state, and neglect the in-plane magnetostatic energy of the Néel walls $[4,25]$. For $\mathrm{Ni} / \mathrm{Fe} / \mathrm{Cu}(001)$, the system studied within this work, the DMI is expected to be much weaker, so that a refined model is needed, which covers the transition from Bloch to Néel walls via a canted wall state due to the competition between DMI energy and in-plane magnetostatic domain wall energy. Here we present a domain spacing model for stripe domain patterns in two-dimensional (2D) ultrathin films, which in contrast to previous approaches also includes the in-plane magnetostatic energy of the domain walls and therefore correctly describes the transition from Bloch to Néel walls in the case of weak DMI. The model considerably extends the work of Kashuba and Pokrovsky [29] as well as similar studies of Won et al. [30-32]. Experimental data obtained by threshold photoemission magnetic circular dichroism photoemission electron microscopy (TP-MCD-PEEM) on $\mathrm{Ni} / \mathrm{Fe}$ bi- and trilayer samples grown epitaxially on $\mathrm{Cu}(001)$ are successfully recovered by the model.

The thermodynamic ground state of a perpendicularly magnetized ferromagnetic film is a lattice of parallel stripe domains, as shown by Yafet and Gyorgy [33]. The magnetization profile of a stripe domain pattern is governed by the competition of domain wall energy comprising exchange, effective anisotropy, and DMI energy and the dipole energy of the stripe lattice favoring a multidomain state due to partial cancellation of the demagnetizing fields of adjacent stripe domains. The effective magnetocrystalline anisotropy is defined as the difference of the uniaxial out-of-plane (OOP) crystalline anisotropy $K_{2}$ and the OOP part of the demagnetizing energy 
such that $K_{2, \text { eff }}=K_{2}-1 / 2 \mu_{0} M_{\mathrm{S}}^{2}$. In the presence of DMI the domain wall energy is lowered [4,19,21,34]. For interfacial DMI in the continuum limit the DMI energy per area is given by $\mathcal{E}_{\mathrm{DMI}}=\mathcal{D}\left[m_{\mathrm{z}} \operatorname{div} \mathbf{m}-(\mathbf{m} \cdot \boldsymbol{\nabla}) m_{\mathrm{z}}\right]$, with $\mathbf{m}$ being the unit magnetization vector and $m_{\mathrm{z}}$ being its $z$ component [21,34]. Here we introduce the $2 \mathrm{D}$ DMI constant $\mathcal{D}=D t=\frac{2 D_{\mathrm{at}}}{a}$, with $D$ being the DMI constant per volume, $t$ being the sample thickness, and $D_{\text {at }}$ being the DMI per atomic bond for a fcc lattice with lattice constant $a$. The ground state of the stripe domain pattern in a thin ferromagnetic film is found by minimizing its total energy per area comprising exchange, anisotropy, DMI, and dipole energy [29-32,35].

Stripe domains in perpendicularly magnetized systems were intensively investigated in the last few decades. Kashuba and Pokrovsky derived a domain spacing model in the 2D limit excluding DMI which predicts an exponential dependence of the stripe domain width on the exchange constant, the effective perpendicular anisotropy $K_{2 \text {,eff }}$, and the square of the saturation magnetic moment per area $\mathcal{M}_{\mathrm{S}}$ [29,35]. Won et al. pointed out that depending on the effective perpendicular anisotropy, the stripe domain phase in ultrathin perpendicularly magnetized films has to be divided into two regimes: the normal stripe phase for sufficiently large effective anisotropy and the spin helix/cycloid phase for very small anisotropy in the vicinity of the spin-reorientation transition (SRT) from OOP to in-plane (IP) magnetization [30,32]. The reason for the existence of the spin helix/cycloid phase is that with decreasing anisotropy the domain walls grow, whereas the stripe domain width decreases, so that neighboring domain walls merge, finally leading to a sinusoidal shape of the domains.

Recently, chiral Néel walls were found by direct imaging of the domain walls in $\mathrm{Fe} / \mathrm{Ni} / \mathrm{Cu}(001)$ and $\mathrm{Co} / \mathrm{Pt}(111)$ using high-resolution magnetic microscopy $[19,20,36]$. Within these works the strength of the DMI is quantified by direct measurement of the domain wall profile. However, this requires a very high spatial and, due to possible domain wall fluctuations, also temporal resolution of the microscope. Here we present an approach to determine the shape of the domain wall and the strength of the DMI solely from the dependence of the stripe domain width on the effective perpendicular anisotropy, demanding thereby much lower spatial resolution than for direct observation of single domain walls. The determination of the shape of a domain wall (achiral Bloch, chiral Néel, or canted transition state) and of the DMI strength is an important step when characterizing heterostructures for future applications in data storage since it is crucial for data retention to have homochiral skyrmionic bubble domains stabilized by a strong DMI.

\section{DOMAIN SPACING MODEL}

In the following calculation of the equilibrium domain width of a stripe domain pattern, stripe domains are assumed to be parallel to the $y$ direction, and the $z$ direction is the OOP direction [see Fig. 1(a)]. As a first step, the domain wall energy has to be derived. For this purpose a generalized collective coordinate approach based on the well-known $q-\Phi$ model $[21,37]$ is applied. The domain wall profile is described by the angle $\theta(x)$ of the unit magnetization vector $\mathbf{m}$ with respect to the $z$ axis and the constant angle $\phi$ of the in-plane projection of m with respect to the $x$ axis. Since the thickness of the sample is much lower than the exchange length, a homogeneous $m_{\mathrm{z}}$ is assumed.

The domain wall profile can be calculated analytically by minimizing the domain wall energy functional comprising exchange, anisotropy, and DMI energy and the OOP part of the demagnetizing energy,

$$
\begin{aligned}
E_{\text {wall }}[\theta(x), \phi]= & \int d x\left[\mathcal{A}\left(\frac{\partial \theta}{\partial x}\right)^{2}+\mathcal{D} \frac{\partial \theta}{\partial x} \cos (\phi)\right. \\
& \left.+\mathcal{K}_{2, \text { eff }} \cos ^{2}(\theta)\right]
\end{aligned}
$$

with the integrated exchange constant $\mathcal{A}=\sum_{i} A_{i} t_{i}$ using the exchange constants $A_{i}$ and thicknesses $t_{i}$ of the individual ferromagnetic layers and the effective perpendicular anisotropy per area $\mathcal{K}_{2 \text {,eff }}[21,34]$. This leads to the wellknown solution $m_{\mathrm{z}}=\tanh (x / l), m_{\mathrm{x}}=\cos (\phi) / \cosh (x / l)$, and $m_{\mathrm{y}}=\sin (\phi) / \cosh (x / l)$, where $\pi l$ is the domain wall width $[21,34]$. The magnetostatic energy of the domain wall is not included in this calculation to retain an analytic solution. According to micromagnetic simulations of Thiaville et al. the domain wall profile including magnetostatics differs from this approximation only at the boundaries of the domain wall, where the in-plane unit magnetization is already below $10^{-3}$ [21]. However, although the deviation of the domain wall profile is not strong, the contribution of the magnetostatic energy to the total domain wall energy can be large and is crucial to describe the transition from Bloch to Néel walls with increasing DMI constant correctly.

Therefore the magnetostatic energy of the domain wall is calculated using the approximated wall profile. The demagnetizing field inside the domain wall can be found by a standard approach based on solving the Poisson equation for the magnetostatic charges induced by the domain wall. This procedure, described in detail in the Supplemental Material [38], leads to the following solution for the magnetostatic energy contribution per wall length:

$$
E_{\mathrm{ms}}=\frac{1}{2} \Omega \cos ^{2}(\phi) k\left(\frac{t}{l}\right),
$$

where $\Omega=\mu_{0} \mathcal{M}_{\mathrm{S}}^{2}$ is the dipole interaction strength using the saturation magnetic moment per area $\mathcal{M}_{\mathrm{S}}$ and $k(t / l)$ is a two-dimensional integral depending on the ratio of sample thickness $t$ and domain wall parameter $l$, which can be calculated numerically for any specific $t / l$. However, for $\mathrm{Fe} / \mathrm{Ni} / \mathrm{Cu}(001)$ the $2 \mathrm{D}$ limit $t / l \rightarrow 0$ can be applied, leading to the known result of $k=2 \ln (2) / \pi \approx 0.44$ [39] (see the Supplemental Material [38]).

By inserting the magnetization profile in Eq. (1) and adding the magnetostatic energy defined in Eq. (2) the domain wall energy per wall length is found to be

$$
E_{\text {wall }}=\frac{2 \mathcal{A}}{l}+2 \mathcal{K}_{2, \text { eff }} l-\pi|\mathcal{D}| \cos (\phi)+\frac{1}{2} k \Omega \cos ^{2}(\phi) .
$$

By minimizing this energy with respect to $l$ and $\phi$ the equilibrium parameters for a single domain wall in infinite 


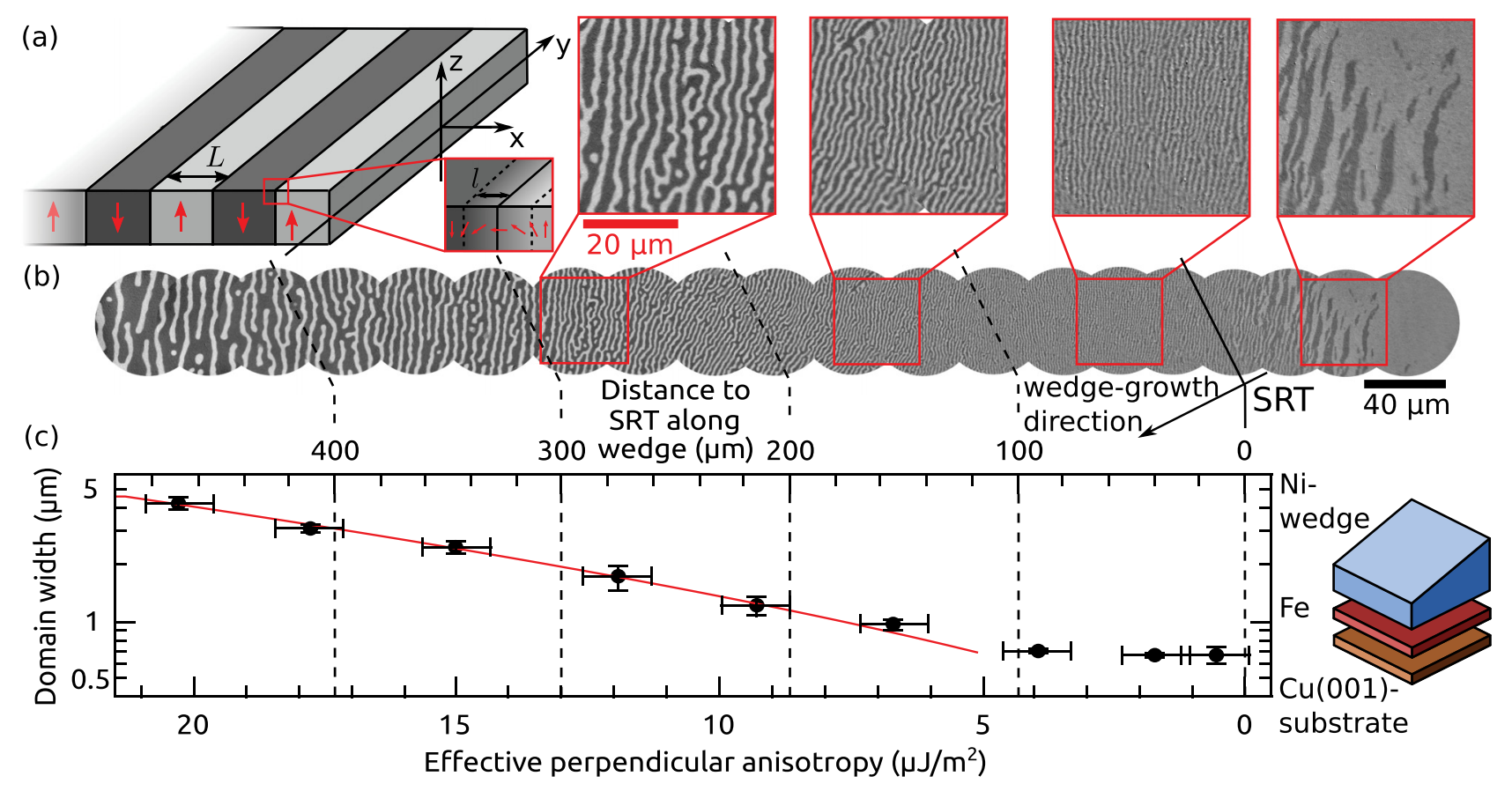

FIG. 1. Domain model applied to the stripe pattern of a Ni/Fe/Cu(001) sample. The coordinate system used for the derivation of the model function and the definition of the domain width $L$ and the domain wall parameter $l$ is sketched in (a). (b) shows a series of TP-MCD-PEEM images across the SRT of a (6-12 ML) Ni/(1.1 ML) Fe/Cu(001) wedge-shaped sample. Above the line scan zoomed images of the domain structure are shown in order to visualize the small domains at the SRT. The domain width is plotted in (c) against the distance to the SRT along the wedge-growth direction. Note that there is an angle of $26^{\circ}$ between the line scan direction and the wedge-growth direction, leading to a scaling factor of 0.9 between the distance to the SRT along the wedge and the distance to the SRT along the line scan. The domain width first decreases exponentially, when approaching the SRT, as long as the sample is in the normal stripe phase. Within the spin cycloid phase for $\mathcal{K}_{2, \text { eff }}<5 \mu \mathrm{J} / \mathrm{m}^{2}$ the domain width converges to a minimum value of (660 \pm 70$) \mathrm{nm}$. A fit with the domain model function is in excellent agreement with the data in the normal stripe phase.

space are obtained as $l=\sqrt{\mathcal{A} / \mathcal{K}_{2, \text { eff }}}$ and [21]

$$
\begin{aligned}
\cos (\phi) & =\frac{\pi \mathcal{D}}{k \Omega} \quad \text { for } \quad \pi|\mathcal{D}|<k \Omega, \\
\phi & =0, \pi \quad \text { for } \quad \pi|\mathcal{D}| \geqslant k \Omega .
\end{aligned}
$$

Using this result, the equilibrium domain wall energy can be written in a more compact way by introducing a domain shape parameter $\Delta$ so that

$$
E_{\text {wall,equ }}=\frac{2 \mathcal{A}}{l}+2 \mathcal{K}_{2, \text { eff }} l-\frac{1}{2} k \Delta \Omega,
$$

where $\Delta$ depends on the magnitude of the DMI constant $\mathcal{D}$ in the following way:

$$
\Delta=\left\{\begin{array}{lll}
0 & \mathcal{D}=0 & \text { Bloch wall, } \\
\left(\frac{\pi \mathcal{D}}{k \Omega}\right)^{2} & \pi|\mathcal{D}|<k \Omega & \text { Canted wall, } \\
\frac{2}{k \Omega}\left(\pi|\mathcal{D}|-\frac{1}{2} k \Omega\right) & \pi|\mathcal{D}| \geqslant k \Omega & \text { Neel wall. }
\end{array}\right.
$$

Note that the domain walls are Bloch walls for $\Delta=0$, canted walls for $0<\Delta<1$, and purely chiral Néel walls for $\Delta \geqslant 1$. Hence $\Delta$ determines the shape of the domain wall.

The total energy of the stripe pattern for zero external field is given by adding Eq. (4) to the dipole energy of the stripe pattern obtained from the work of Kashuba and Pokrovsky [29],

$$
\mathcal{E}_{\text {tot }}=\frac{2 \mathcal{A}}{L l}+2 \mathcal{K}_{2, \text { eff }} \frac{l}{L}-\frac{1}{2} \frac{k \Delta \Omega}{L}-\frac{\Omega}{\pi L} \ln \left(\frac{2 L}{\pi l}\right),
$$

where $L$ is the stripe domain width as illustrated in Fig. 1(a).

The equilibrium state of the domain pattern is determined by minimizing Eq. (6) with respect to $L$ and $l[29,31,32]$, resulting in

$$
\begin{aligned}
& l_{\mathrm{eq}}=\sqrt{\frac{\mathcal{A}}{\mathcal{K}_{2, \mathrm{eff}}}+\frac{\Omega^{2}}{16 \pi^{2} \mathcal{K}_{2, \mathrm{eff}}^{2}}}-\frac{\Omega}{4 \pi \mathcal{K}_{2, \mathrm{eff}}}, \\
& L_{\mathrm{eq}, 0}=\frac{\pi}{2} l_{\mathrm{eq}} \exp \left(\frac{4 \pi \mathcal{A}}{\Omega l_{\mathrm{eq}}}-\frac{\pi k}{2} \Delta\right),
\end{aligned}
$$

where $L_{\text {eq, } 0}$ is the equilibrium domain width.

Note that the domain model is subject to some restrictions originating from assumptions made during the calculation. This model relies on a full OOP magnetization within the stripe domains, which is no longer fulfilled in close vicinity to the SRT, where the magnetization is canted with respect to the sample normal [40]. When approaching the SRT the domain width becomes comparable to the domain wall width, and the tanh-like domain wall profile is replaced by a spin cycloid structure [30,32]. The domain model is not valid in the spin cycloid phase due to the different domain wall profiles and due to the fact that the long-range part of the dipole energy of the domain walls was neglected in the calculation of the dipole 
energy of the stripe pattern [29], whereas the short-range part of the magnetostatic wall energy is fully included in the model. Thus the domain model is valid only in the normal stripe phase.

In the following the domain model will be applied to measurements of the domain width in the normal stripe phase of several samples based on $\mathrm{Ni} / \mathrm{Fe} / \mathrm{Cu}(001)$. The application of the model allows the determination of the domain shape parameter $\Delta$ and the magnitude of the DMI constant $|\mathcal{D}|$ in these samples.

\section{APPLICATION OF THE DOMAIN SPACING MODEL: METHOD}

The samples are grown on a single-crystalline $\mathrm{Cu}(001)$ substrate with a miscut angle less than $0.1^{\circ}$. Before sample growth the substrate is prepared by several cycles of sputtering with argon ions of $0.8-1.2 \mathrm{keV}$ energy and subsequent annealing at $820 \mathrm{~K}$ for $30 \mathrm{~min}$. All samples are grown at room temperature by molecular beam epitaxy (MBE) at a base pressure of $3 \times 10^{-10}$ mbar using electron beam evaporators. The pressure during growth does not exceed $8 \times 10^{-10}$ mbar. Layer-by-layer growth of the iron and nickel layers is monitored by reflection high-energy electron diffraction oscillations as well as measurement of the atomic flux so that an exact determination of the thickness of each layer with an accuracy of 0.1 monolayer (ML) is achieved. The thickness of the platinum overlayer on some samples was measured using a calibrated quartz microbalance. A movable shadow mask allows the growth of wedge-shaped samples.

Subsequent to the growth, the sample is immediately transferred into the PEEM chamber with a base pressure of $4 \times 10^{-11}$ mbar without leaving the UHV environment. The domain structure of the samples is investigated by TP-MCDPEEM, which is described in detail elsewhere [41]. A coil can be used to apply small out-of-plane magnetic fields of up to $0.03 \mathrm{~T}$ to the sample and to compensate a small residual field of $1 \times 10^{-4} \mathrm{~T}$ inside the chamber.

For each sample a line scan is taken in the stripe domain phase in zero magnetic field starting from large stripe domains with a domain width of several micrometers and ending in the in-plane phase, where either no contrast or very large irregular in-plane domains are visible [see Fig. 1(b)]. The stripe domain width decreases with decreasing effective perpendicular magnetic anisotropy and reaches a minimum at the SRT, where $\mathcal{K}_{2 \text {,eff }}=0$. The domain width is measured along the line scan and plotted versus the distance to the SRT [see the example in Fig. 1(c)]. Every data point in this graph is obtained from a single image of the line scan by averaging the domain width along several lines in the image while taking equal numbers of black and white domains for each average. The error bar is the standard deviation of the measured data along all lines.

Since the wedge is grown by linear motion of the shadow mask, the distance to the SRT along the wedge growth direction is proportional to the change of the effective perpendicular anisotropy per area as long as the thickness of the nickel layer is smaller than $3.5 \mathrm{~nm}$ so that the strain due to epitaxial growth causing the perpendicular anisotropy is not significantly relaxed [42]. Note that the nickel thickness is always larger than $3 \mathrm{ML}$, so that the surface or interface parts of the anisotropy of the nickel layer are constant, and only the volume contribution changes. Given that $\mathcal{K}_{2 \text {,eff }}=0$ at the SRT, the absolute effective perpendicular anisotropy along the line scan can be calculated as $\mathcal{K}_{2, \text { eff }}=\Delta \mathcal{K}_{2, \text { eff,ML }} s x_{\mathrm{SRT}}$, where $\Delta \mathcal{K}_{2, \text { eff,ML }}$ is the volume part of the effective twofold anisotropy per area of $1 \mathrm{ML}$ of $\mathrm{Ni}, s$ is the slope of the wedge in units of $\mathrm{ML} / \mathrm{m}$, and $x_{\mathrm{SRT}}$ is the distance to the SRT along the wedge-growth direction.

The effective twofold anisotropy per area of $1 \mathrm{ML}$ of $\mathrm{Ni}$ can be derived from the well-known volume anisotropy of $\mathrm{Ni}$ on $\mathrm{Cu}(001), K_{\mathrm{V}, \mathrm{Ni}}=30 \mu \mathrm{eV} /$ atom [43-45], and is given by $\Delta \mathcal{K}_{2, \text { eff, ML }}=\left(K_{\mathrm{V}, \mathrm{Ni}}-1 / 2 \mu_{0} M_{\mathrm{S}}^{2}\right) a / 2=50.6 \mu \mathrm{J} / \mathrm{m}^{2} \mathrm{ML}$ using a monolayer thickness of $a / 2=1.8 \AA$ and the saturation magnetic moment of nickel $\mu_{\mathrm{Ni}}=0.57 \mu_{\mathrm{B}}[30,31,44,46,47]$.

With the knowledge of the dependence of the domain width on the effective perpendicular anisotropy the domain spacing model described in Eq. (8) can be fitted to the measured data. The fit function depends on three model parameters: the integrated exchange constant $\mathcal{A}$, the dipole interaction strength $\Omega$, and the domain shape parameter $\Delta$, containing the DMI constant. In order to obtain better fit results it is useful to fix one of the parameters. The dipole interaction strength $\Omega=\mu_{0} \mathcal{M}_{\mathrm{S}}^{2}$ is most suitable for this purpose since it is the only quantity for which reliable literature values exist. Note that the thickness of the nickel layer changes at maximum by half a monolayer during all line scans shown within this work, corresponding to a maximum change of $6 \%$ of the dipole interaction strength $\Omega$ and a $3 \%$ change of the exchange constant $\mathcal{A}$, so that $\Omega$ and $\mathcal{A}$ can be assumed to be constants in the fit model function. The saturation magnetic moment per area can be determined from $\mu_{\mathrm{Ni}}=0.57 \mu_{\mathrm{B}}$ for nickel $[44,46,47]$ and from the bulk value $\mu_{\mathrm{Fe}}=2.1 \mu_{\mathrm{B}}$ for iron using $\mathcal{M}_{\mathrm{S}}=\left(\mu_{\mathrm{Ni}} d_{\mathrm{Ni}}+\mu_{\mathrm{Fe}} d_{\mathrm{Fe}}\right) / a_{\mathrm{p}}^{2}$, with the in-plane lattice constant $a_{\mathrm{p}}=2.55 \AA$ of $\mathrm{Cu}$ due to pseudomorphic growth and the thicknesses of the nickel and iron layers at the SRT $d_{\mathrm{Ni}}$ and $d_{\mathrm{Fe}}$ in units of monolayers. The integrated exchange constant $\mathcal{A}$ can be estimated as $\mathcal{A}=A_{\mathrm{Ni}} d_{\mathrm{Ni}} a / 2+A_{\mathrm{Fe}} d_{\mathrm{Fe}} a / 2$, with $A_{\mathrm{Ni}}=0.8 \times 10^{-11} \mathrm{~J} / \mathrm{m}$ [48] and the bulk value of $A_{\mathrm{Fe}}=$ $1.9 \times 10^{-11} \mathrm{~J} / \mathrm{m}$ for iron. Note that in the fits in the next section the exchange constant $\mathcal{A}$ is a free parameter; the estimate formula for $\mathcal{A}$ given here is used only for cross-checking the fit results. For the calculation of the error bars in the next section an error of $20 \%$ is assumed for $\Omega$, and an error of $10 \%$ is assumed for the volume part of the anisotropy of nickel.

\section{APPLICATION OF THE DOMAIN SPACING MODEL: RESULTS}

A fit of the measured domain width with the domain model function assuming a fixed value of $\Omega=1.42 \mathrm{pJ} / \mathrm{m}$ is shown in Fig. 1(c) for a Ni/Fe/Cu(001) sample with $d_{\mathrm{Ni}}=9 \mathrm{ML}$ and $d_{\mathrm{Fe}}=1.1 \mathrm{ML}$ at the SRT. Note that the spin cycloid phase has to be excluded from the fit according to the previous discussion. The two phases can be distinguished easily by a kink in the logarithmic plot shown in Fig. 2(a). The fit represents the data very well in the normal stripe phase and results in a $2 \mathrm{D}$ exchange constant of $\mathcal{A}=(90 \pm 40) \mathrm{meV}$ and in a domain shape parameter $\Delta=1.7 \pm 0.8$, indicating that the domain walls are in a pure Néel wall state, as already found 

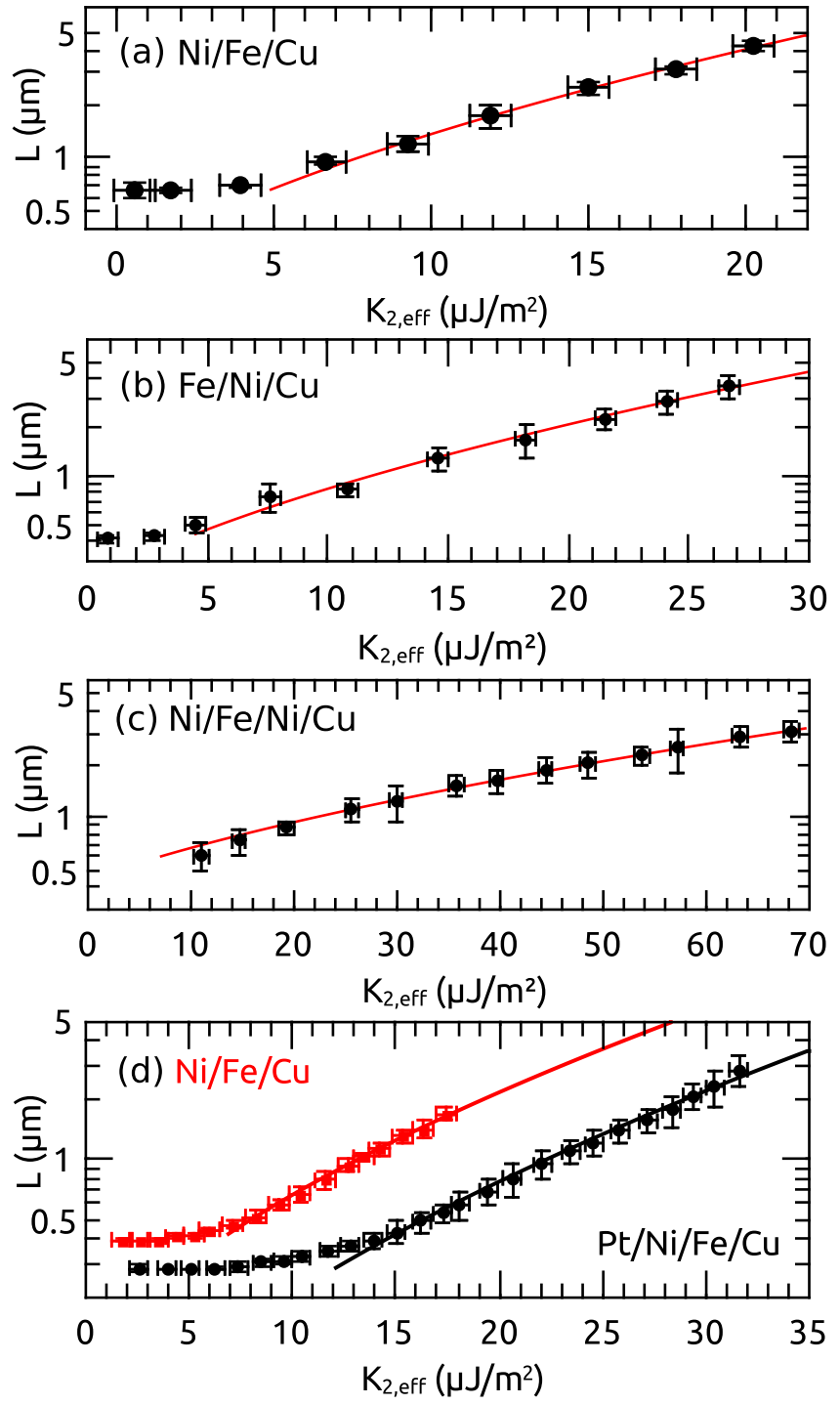

FIG. 2. Domain width versus effective anisotropy for various sample configurations. The measured domain width deduced from an analysis of images across the SRT is plotted logarithmically as black dots, and the corresponding fit with the domain model function is shown as a red line. (a) shows the domain width for the same line scan as in Fig. 1. In the logarithmic plot the transition from the normal stripe to the spin cycloid phase is clearly visible as a kink. Data within the spin cycloid phase are excluded from each fit. In (b) (1-3 ML) $\mathrm{Fe} /(6-12 \mathrm{ML}) \mathrm{Ni} / \mathrm{Cu}(001)$ is investigated; (c) is a test of the domain model with a symmetric stack of (4-14 ML) Ni/(1-3 ML) Fe/(9 ML) $\mathrm{Ni} / \mathrm{Cu}(001)$, and in (d) the effect of a platinum overlayer on the domain structure is examined on (6-12 ML) Ni/(1 ML) $\mathrm{Fe} / \mathrm{Cu}(001)$ with one half covered by $0.4 \mathrm{~nm}$ of Pt. The black dots represent data on the Pt-covered side, and the red squares represent the half without Pt.

by Chen et al. [19]. The DMI constant is calculated according to Eq. (5), leading to $|\mathcal{D}|=(0.28 \pm 0.14) \mathrm{pJ} / \mathrm{m}$ or, in atomic form, $\left|D_{\text {at }}\right|=(0.3 \pm 0.14) \mathrm{meV} /$ atom. This result is in reasonable agreement with the estimate of $0.12-0.17 \mathrm{meV} /$ atom obtained by Chen et al. in a study of the domain walls in $\mathrm{Fe} / \mathrm{Ni} / \mathrm{Cu}(001)$ [19]. The same procedure is applied to a $\mathrm{Fe} / \mathrm{Ni} / \mathrm{Cu}(001)$ sample with $d_{\mathrm{Ni}}=9 \mathrm{ML}$ and $d_{\mathrm{Fe}}=1.4 \mathrm{ML}$ at the SRT. Due to the different stack orders the sign of the
DMI constant is reversed compared to $\mathrm{Ni} / \mathrm{Fe} / \mathrm{Cu}(001)$, but the magnitude should be the same. The measured domain width versus effective perpendicular anisotropy and a fit assuming a fixed $\Omega=1.66 \mathrm{pJ} / \mathrm{m}$ are shown in Fig. 2(b). From the fit $\mathcal{A}=(90 \pm 40) \mathrm{meV}$ and a DMI constant with the magnitude $|\mathcal{D}|=(0.30 \pm 0.12) \mathrm{pJ} / \mathrm{m}$ are obtained, which is again in good agreement with the value for $\mathrm{Ni} / \mathrm{Fe} / \mathrm{Cu}(001)$.

In order to test the validity of the DMI constants obtained from the fits, the model is also applied to a stripe-forming system without DMI. A symmetric $\mathrm{Ni} / \mathrm{Fe} / \mathrm{Ni} / \mathrm{Cu}(001)$ sample should have a negligible DMI constant since the contributions of $\mathrm{FeNi}$ and $\mathrm{NiFe}$ interfaces cancel each other. The domain width of a (4-14 ML) Ni/(1-3 ML) Fe/(9 ML) Ni/Cu(001) sample with a total nickel thickness $d_{\mathrm{Ni}}=21 \mathrm{ML}$ and $d_{\mathrm{Fe}}=$ $0.8 \mathrm{ML}$ at the SRT is plotted in Fig. 2(c). A fit with the domain model using a fixed $\Omega$ results in $\mathcal{A}=(190 \pm 80) \mathrm{meV}$ and $\Delta=0.1 \pm 0.3$, indicating that the domain walls are Bloch walls and that the DMI constant is zero within the error bars. This is clear proof that the domain model delivers reasonable values for the DMI constants.

Next, the domain spacing model is subjected to a different sanity check. By adding an overlayer of a heavy transition metal with large spin-orbit coupling on top of $\mathrm{Ni} / \mathrm{Fe} / \mathrm{Cu}(001)$ the strength of the DMI should be considerably enhanced. A (6-12 ML) $\mathrm{Ni} /(1 \mathrm{ML}) \mathrm{Fe} / \mathrm{Cu}(001)$ sample is prepared, and half of the sample is covered with $0.4 \mathrm{~nm}$ of platinum. The domain width on the pure $\mathrm{Ni} / \mathrm{Fe} / \mathrm{Cu}(001)$ sample is shown as red squares, and that on the Pt-covered part is shown as black dots in Fig. 2(d). Both data sets are fitted with fixed $\Omega$, adding a proximity polarization of $0.2 \mu_{\mathrm{B}}$ per platinum atom at the interface [49] to the total magnetic moment for the Pt-covered part of the sample. Without the platinum $\left|\mathcal{D}_{\mathrm{Ni}}\right|=(0.38 \pm 0.14) \mathrm{pJ} / \mathrm{m}$, whereas with the platinum overlayer the DMI constant is strongly increased to $\left|\mathcal{D}_{\text {Pt }}\right|=(0.6 \pm 0.2) \mathrm{pJ} / \mathrm{m}$. By adding a material with strong spin-orbit coupling on top of $\mathrm{Ni} / \mathrm{Fe} / \mathrm{Cu}(001)$ it is hence, as expected, possible to considerably enhance the strength of the DMI.

\section{PHASE DIAGRAMS}

Following the previous discussion it is clear that the DMI in $\mathrm{Ni} / \mathrm{Fe} / \mathrm{Cu}(001)$-based systems is sufficiently strong to stabilize a homochiral stripe domain phase at zero magnetic field. In such systems homochiral, so-called skyrmionic bubble domains are expected to exist in a certain range of externally applied OOP magnetic fields [20,50,51]. In order to investigate the bubble domain phase, phase diagrams of a Ni/Fe/Cu(001) sample and a Pt-covered $\mathrm{Ni} / \mathrm{Fe} / \mathrm{Cu}(001)$ sample are presented in this section. A similar phase diagram has already been discussed in previous works by Saratz et al. [52,53] for $\mathrm{Fe} / \mathrm{Cu}(001)$, a system with no DMI. Here we report phase diagrams of stripe-forming systems with significant DMI and show that scaling laws obtained before $[52,53]$ are still valid and that a universal phase diagram of stripe-forming systems can be obtained independent of the specific sample type.

The phase diagrams are obtained using the following procedure: First, a line scan of the stripe domain pattern in zero field is recorded, where zero field is defined by equal width of up and down magnetized domains. Then the field 

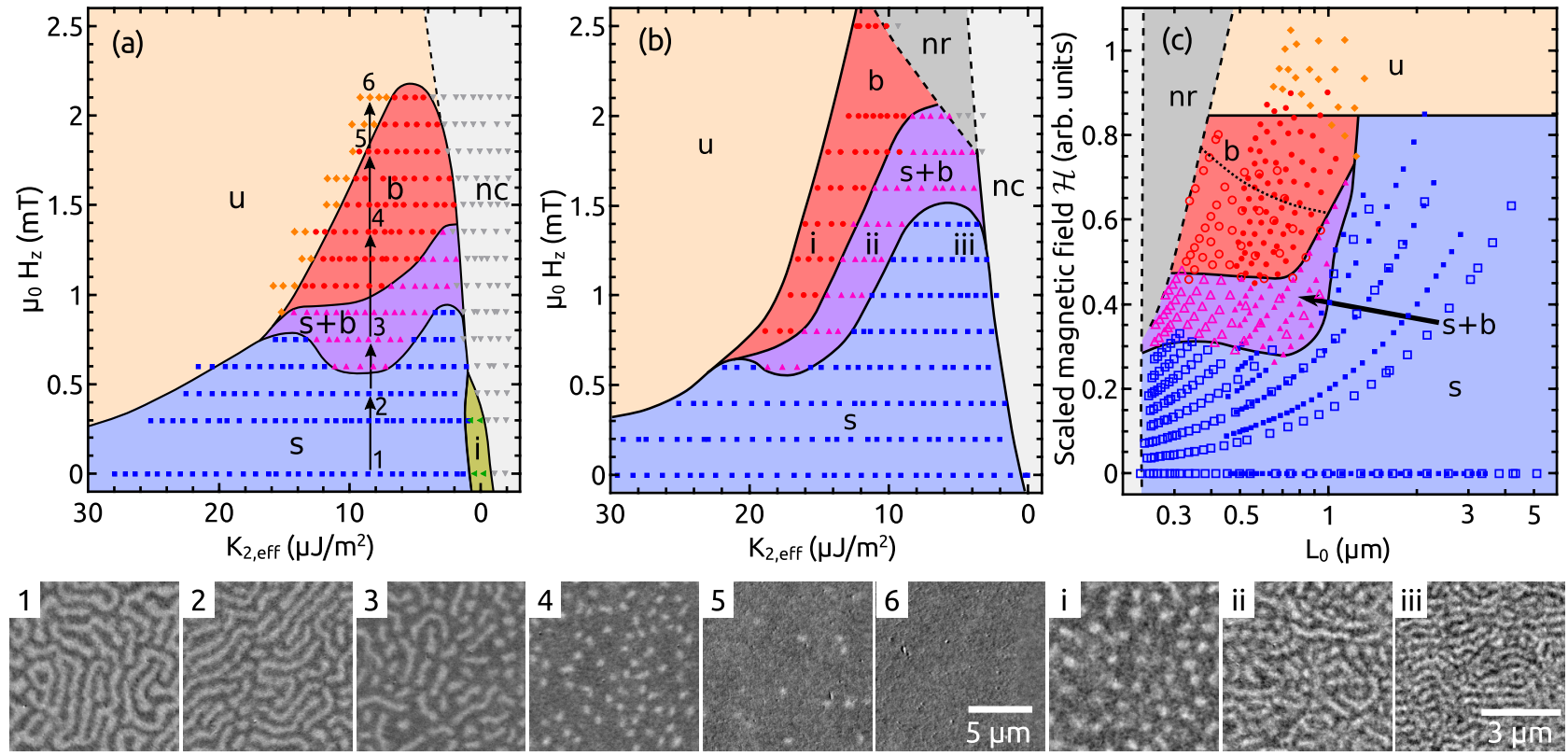

FIG. 3. Phase diagrams of two samples based on $\mathrm{Ni} / \mathrm{Fe} / \mathrm{Cu}(001)$. (a) shows an OOP magnetic field versus perpendicular anisotropy phase diagram of a $(6.5-11.5 \mathrm{ML}) \mathrm{Ni} /(1.2 \mathrm{ML}) \mathrm{Fe} / \mathrm{Cu}(001)$ sample with $d_{\mathrm{Ni}}=10 \mathrm{ML}$ at the SRT. (b) shows the same phase diagram for $(0.4 \mathrm{~nm})$ $\mathrm{Pt} /(6-12 \mathrm{ML}) \mathrm{Ni} /(1.1 \mathrm{ML}) \mathrm{Fe} / \mathrm{Cu}(001)$ with $d_{\mathrm{Ni}}=9 \mathrm{ML}$ at the SRT. In each of the phase diagrams several phases consisting of different magnetic textures appear. The stripe domain phase (s), a mixed phase of stripes and bubble domains ( $\mathrm{s}+\mathrm{b}$ ), the bubble domain phase (b), and the uniform state $(\mathrm{u})$ at large magnetic fields are indicated. Around zero perpendicular anisotropy in-plane domains or mixtures of in-plane domains and stripes (i) can appear. At negative perpendicular anisotropy no visible domain contrast (nc) is obtained. Parts of the phase diagram cannot be resolved due to the lack of resolution of the PEEM in a large OOP magnetic field (nr). Images 1 to 6 show the stripe-to-bubble transition of the domain pattern with increasing magnetic field, and images (i) to (iii) show the transition from bubbles to stripes for decreasing anisotropy. In (c) a combined, universal phase diagram of both samples is plotted versus the stripe domain width at zero field $L_{0}$ with the magnetic field axis scaled by a factor of $\frac{L_{0}}{\mathcal{M}_{\mathrm{S}}}$. Open symbols correspond to the Pt-covered sample, and closed symbols correspond to the $\mathrm{Ni} / \mathrm{Fe} / \mathrm{Cu}(001)$ sample. The dotted line inside the bubble domain phase separates closely packed bubble domains [comparable to image 4 or (i)] at lower magnetic field from low-density bubble domains at higher field.

is increased in a small step. After keeping a waiting time of several minutes in order to allow equilibration of the domain pattern the next line scan is acquired. The last two steps are repeated until saturation of the sample is observed. The distance to the SRT of each individual image in the line scans is converted to the effective perpendicular anisotropy $\mathcal{K}_{2 \text {,eff }}$ as described in Sec. III and plotted versus the OOP magnetic field in Fig. 3(a) for the Ni/Fe/Cu(001) sample and in Fig. 3(b) for the $\mathrm{Pt} / \mathrm{Ni} / \mathrm{Fe} / \mathrm{Cu}(001)$ sample. The state of the domain pattern at each point is given by its color. Furthermore, approximated phase boundaries are plotted as guides to the eye in each phase diagram.

Depending on the effective perpendicular anisotropy two regions can be distinguished in both phase diagrams. In the first region in the vicinity of the SRT, where the domain width in zero magnetic field is smaller than $1 \mu \mathrm{m}$, bubble domains are observed. Starting from the stripe phase (labeled s) at zero field, the stripe domains begin to break apart at a certain field, leading to a mixed phase of stripes and bubble domains $(\mathrm{s}+\mathrm{b})$ until at higher magnetic fields a pure bubble domain state (b) can be reached. Further increasing the magnetic field leads to bubble domains with low density at the edge of the bubble domain phase and, finally, to a uniform state (u) as the bubble domains vanish. This transition is visualized by images 1 to 6 at the bottom of Fig. 3. In the second region for domain width larger than $1 \mu \mathrm{m}$ no bubble domains are observed when increasing the magnetic field, but a metastable stripe state persists up to the saturation of the sample. Fission of stripe domains with a large domain width requires overcoming a large energy barrier, so that the stripe domains cannot split in an experimentally accessible time scale [52,53]. Around zero perpendicular anisotropy we observe in-plane domains or mixtures of in-plane domains and stripes in a small region near the SRT labeled (i). For larger negative perpendicular anisotropy a uniformly in-plane magnetized state with no contrast is obtained (nc). The saturation field for the $\mathrm{Ni} / \mathrm{Fe} / \mathrm{Cu}(001)$ sample is around $2.1 \mathrm{mT}$, whereas the platinum-covered sample is still not saturated at $2.5 \mathrm{mT}$. Note that applying magnetic fields of this magnitude in a PEEM leads to considerable distortions of the image, so that we are neither able to resolve the small bubble domains in the Pt-covered sample for larger magnetic fields (nr) nor able to measure the saturation field of the Pt-covered sample. The minimum size of the stripe domains at the SRT is $460 \pm 25 \mathrm{~nm}$ for the $\mathrm{Ni} / \mathrm{Fe} / \mathrm{Cu}(001)$ sample with a minimum bubble domain diameter of $430 \pm 50 \mathrm{~nm}$ and $220 \pm 20 \mathrm{~nm}$ for the Pt-covered sample with a minimum resolvable bubble domain diameter of $260 \pm 30 \mathrm{~nm}$.

If the domain pattern is investigated with respect to the effective anisotropy in a constant, sufficiently large OOP magnetic field, first, a transition from the saturated state at large anisotropy to the bubble domain phase is observed. 
With decreasing anisotropy the bubble domains merge, and finally, a stripe domain phase is formed. This transition is visualized in images (i) to (iii) at the bottom of Fig. 3 . Note that decreasing the effective anisotropy is equivalent to heating the sample [54], so that in this case the same sequence of pattern transformations is observed.

The phase diagrams of stripe-forming systems with perpendicular anisotropy show some general scaling laws, which can already partly be derived from the domain spacing model (see the Supplemental Material [38]). The critical field $H_{\mathrm{c}}$, above which the ground state of the sample is uniform, is proportional to

$$
H_{\mathrm{c}} \propto \frac{\mathcal{M}_{\mathrm{S}}}{L_{0}},
$$

where $L_{0}$ is the measured domain width in zero applied field (see the Supplemental Material) [29,35,38]. Even though this result was derived for a stripe domain pattern and not for bubble domains, the proportionality of the saturation field to $\frac{\mathcal{M}_{\mathrm{s}}}{L_{0}}$ is also applicable for bubble domains, as shown by Saratz et al. using numerical calculations [52]. Furthermore, it was shown that the stripe-to-bubble transition should take place at $H_{\mathrm{b}}=0.4 H_{\mathrm{c}}$ at any anisotropy [52].

These scaling laws are tested in Fig. 3(c), where a scaled phase diagram comprising both samples is plotted. On the $x$ axis the domain width at zero field $L_{0}$ is shown, and on the $y$ axis a dimensionless scaled magnetic field according to $\mathcal{H}=\frac{L_{0}}{\mathcal{M}_{\mathrm{s}}} H_{\mathrm{z}}$ is shown. It can be immediately obtained from the graph that the scaled stripe-to-bubble transition field and the scaled saturation field of both samples are independent of the zero-field domain width and independent of the sample type. Hence the scaling laws proposing a proportionality of the saturation field $H_{\text {sat }}$ and the stripe-to-bubble transition field $H_{\mathrm{b}}$ to $\mathcal{M}_{\mathrm{S}} / L_{0}$ obtained from the domain model and the work of Saratz et al. [52,53] are valid for both samples independent of the DMI strength as long as the domain wall energy stays positive. Hence DMI affects only the domain width at zero field and, after proper scaling, not the behavior in a magnetic field. The unscaled saturation field $H_{\text {sat }}$ of course increases strongly proportionally to $1 / L_{0}$ with increasing DMI constant.

From the graph the saturation field $\mathcal{H}_{\text {sat }}=0.8 \pm 0.1$ and the stripe-to-bubble transition field $\mathcal{H}_{\mathrm{b}}=0.29 \pm 0.03$ can be estimated. Note that the saturation field is not equal to the critical field $\mathcal{H}_{\mathrm{c}}=\frac{4}{\exp (2)}$ determined for bubble domains in thermal equilibrium by Saratz et al. [52,53] since metastable bubble domains with low density are observed above the dotted line in Fig. 3(c), which might be caused by pinning at structural features of the substrate [53]. A better estimate for the critical field from the graph in Fig. 3(c) is the position of the dotted transition line from closely packed to low-density bubble domains at $\mathcal{H}_{\mathrm{cb}, \text { ldb }} \approx 0.7 \pm 0.1$, above which the bubble domains are not the ground state anymore and start to decay. The ratio of the stripe-to-bubble transition field to the saturation field is $0.36 \pm 0.08$, which is in reasonable agreement with the theoretical expectation value of 0.41 .

\section{CONCLUSION}

In conclusion a domain spacing model for the normal stripe domain phase was developed for ultrathin perpendicularly magnetized ferromagnetic films based on the $q-\Phi$ domain wall model including DMI and in-plane magnetostatic energy of the domain walls. By minimizing the total energy of the stripe domain pattern a model function for the domain width dependent on the magnetic properties of the sample was obtained. The model was successfully applied to several sample structures based on $\mathrm{Ni} / \mathrm{Fe} / \mathrm{Cu}(001)$ by fitting the domain width plotted versus the effective perpendicular anisotropy. By using this method the shape of the domain walls and the magnitude of the DMI constant could be determined for several samples based on $\mathrm{Ni} / \mathrm{Fe} / \mathrm{Cu}(001)$. For $\mathrm{Ni} / \mathrm{Fe} / \mathrm{Cu}(001)$ and $\mathrm{Fe} / \mathrm{Ni} / \mathrm{Cu}(001)$ the fit revealed chiral Néel walls and a DMI constant of $0.3 \mathrm{meV} /$ atom. As a proof of concept the model was applied to a symmetric $\mathrm{Ni} / \mathrm{Fe} / \mathrm{Ni}$ stack delivering zero DMI and Bloch walls, and for a Pt-covered Ni/Fe/Cu(001) sample a strongly enhanced DMI constant was found, as expected. Our model can therefore be used to characterize perpendicularly magnetized material systems regarding DMI strength and other material parameters. The model is valid for any strength of DMI, including weak DMI in the transition region between Bloch and Néel walls and large DMI, as long as the domain wall energy stays positive. Furthermore, phase diagrams for two different samples were obtained in magnetic-field-anisotropy space. By rescaling the magnetic field it was possible to combine the two phase diagrams into a universal phase diagram for a perpendicularly magnetized stripe-forming system.
[1] X. Z. Yu, N. Kanazawa, W. Z. Zhang, T. Nagai, T. Hara, K. Kimoto, Y. Matsui, Y. Onose, and Y. Tokura, Nat. Commun. 3, 988 (2012).

[2] A. Fert, V. Cros, and J. Sampaio, Nat. Nanotechnol. 8, 152 (2013).

[3] J. Iwasaki, M. Mochizuki, and N. Nagaosa, Nat. Nanotechnol. 8, 742 (2013).

[4] S. Woo, K. Litzius, B. Kruger, M. Im, L. Caretta, K. Richter, M. Mann, A. Krone, R. M. Reeve, M. Weigand, P. Agrawal, I. Lemesh, M. Mawass, P. Fischer, M. Kläui, and G. S. D. Beach, Nat. Mater. 15, 501 (2016).

[5] I. Dzyaloshinsky, J. Phys. Chem. Solids 4, 241 (1958).
[6] T. Moriya, Phys. Rev. 120, 91 (1960).

[7] A. Fert and P. M. Levy, Phys. Rev. Lett. 44, 1538 (1980).

[8] A. Neubauer, C. Pfleiderer, B. Binz, A. Rosch, R. Ritz, P. G. Niklowitz, and P. Böni, Phys. Rev. Lett. 102, 186602 (2009).

[9] W. Münzer, A. Neubauer, T. Adams, S. Mühlbauer, C. Franz, F. Jonietz, R. Georgii, P. Böni, B. Pedersen, M. Schmidt, A. Rosch, and C. Pfleiderer, Phys. Rev. B 81, 041203(R) (2010).

[10] M. Uchida, Y. Onose, Y. Matsui, and Y. Tokura, Science 311, 359 (2006)

[11] X. Z. Yu, Y. Onose, N. Kanazawa, J. H. Park, J. H. Han, Y. Matsui, N. Nagaosa, and Y. Tokura, Nature (London) 465, 901 (2010). 
[12] S. Mühlbauer, B. Binz, F. Jonietz, C. Pfleiderer, A. Rosch, A. Neubauer, R. Georgii, and P. Böni, Science 323, 915 (2009).

[13] M. Bode, M. Heide, M. K. von Bergmann, M. P. Ferriani, M. S. Heinze, M. G. Bihlmayer, M. A. Kubetzka, M. O. Pietzsch, M. S. Blügel, and M. R. Wiesendanger, Nature (London) 447, 190 (2007).

[14] P. Ferriani, K. von Bergmann, E. Y. Vedmedenko, S. Heinze, M. Bode, M. Heide, G. Bihlmayer, S. Blügel, and R. Wiesendanger, Phys. Rev. Lett. 101, 027201 (2008).

[15] S. Heinze, K. von Bergmann, M. Menzel, J. Brede, A. Kubetzka, R. Wiesendanger, G. Bihlmayer, and S. Blügel, Nat. Phys. 7, 713 (2011).

[16] A. Hrabec, N. A. Porter, A. Wells, M. J. Benitez, G. Burnell, S. McVitie, D. McGrouther, T. A. Moore, and C. H. Marrows, Phys. Rev. B 90, 020402 (2014).

[17] S.-G. Je, D.-H. Kim, S.-C. Yoo, B.-C. Min, K.-J. Lee, and S.-B. Choe, Phys. Rev. B 88, 214401 (2013).

[18] M. J. Benitez, A. Hrabec, A. P. Mihai, T. A. Moore, G. Burnell, D. McGrouther, C. H. Marrows, and S. McVitie, Nat. Commun. 6, 8957 (2015).

[19] G. Chen, J. Zhu, A. Quesada, J. Li, A. T. N'Diaye, Y. Huo, T. P. Ma, Y. Chen, H. Y. Kwon, C. Won, Z. Q. Qiu, A. K. Schmid, and Y. Z. Wu, Phys. Rev. Lett. 110, 177204 (2013).

[20] G. Chen, A. Mascaraque, A. T. N'Diaye, and A. K. Schmid, Appl. Phys. Lett. 106, 242404 (2015).

[21] A. Thiaville, S. Rohart, E. Jué, and V. C. A. Fert, Europhys. Lett. 100, 57002 (2012).

[22] K.-S. Ryu, L. Thomas, S.-H. Yang, and S. Parkin, Nat. Nanotechnol. 8, 527 (2013).

[23] S. Emori, U. Bauer, S.-M. Ahn, E. Martinez, and G. S. D. Beach, Nat. Mater. 12, 611 (2013).

[24] C. Moreau-Luchaire, C. Moutafis, N. Reyren, J. Sampaio, C. A. F. Vaz, N. Van Horne, K. Bouzehouane, K. Garcia, C. Deranlot, P. Warnicke, P. Wohlhüter, J.-M. George, M. Weigand, J. Raabe, V. Cros, and A. Fert, Nat. Nanotechnol. 11, 444 (2016).

[25] M. Schott, A. Bernand-Mantel, L. Ranno, S. Pizzini, J. Vogel, H. Béa, C. Baraduc, S. Auffret, G. Gaudin, and D. Givord, Nano Lett. 17, 3006 (2017).

[26] W. Jiang, P. Upadhyaya, W. Zhang, G. Yu, M. B. Jungfleisch, F. Y. Fradin, J. E. Pearson, Y. Tserkovnyak, K. L. Wang, O. Heinonen, S. G. E. te Velthuis, and A. Hoffmann, Science 349, 283 (2015).

[27] W. Jiang, X. Zhang, G. Yu, W. Zhang, X. Wang, M. B. Jungfleisch, J. E. Pearson, X. Cheng, O. Heinonen, K. L. Wang, Y. Zhou, A. Hoffmann, and S. G. E. te Velthuis, Nat. Phys. 13, 162 (2017).

[28] I. Lemesh, F. Büttner, and G. S. D. Beach, Phys. Rev. B 95, 174423 (2017).

[29] A. B. Kashuba and V. L. Pokrovsky, Phys. Rev. B 48, 10335 (1993).
[30] C. Won, Y. Z. Wu, J. Choi, W. Kim, A. Scholl, A. Doran, T. Owens, J. Wu, X. F. Jin, H. W. Zhao, and Z. Q. Qiu, Phys. Rev. B 71, 224429 (2005).

[31] Y. Z. Wu, C. Won, A. Scholl, A. Doran, H. W. Zhao, X. F. Jin, and Z. Q. Qiu, Phys. Rev. Lett. 93, 117205 (2004).

[32] H. Y. Kwon, S. S. Hong, J. H. Seok, K. M. Bu, Y. Z. Wu, Z. Q. Qiu, and C. Won, J. Magn. Magn. Mater. 322, 2742 (2010).

[33] Y. Yafet and E. M. Gyorgy, Phys. Rev. B 38, 9145 (1988).

[34] M. Heide, G. Bihlmayer, and S. Blügel, Phys. Rev. B 78, 140403 (2008).

[35] A. Kashuba and V. L. Pokrovsky, Phys. Rev. Lett. 70, 3155 (1993).

[36] E. C. Corredor, S. Kuhrau, F. Kloodt-Twesten, R. Frömter, and H. P. Oepen, Phys. Rev. B 96, 060410 (2017).

[37] N. L. Schryer and L. R. Walker, J. Appl. Phys. 45, 5406 (1974).

[38] See Supplemental Material at http://link.aps.org/supplemental/ 10.1103/PhysRevB.96.144408 for the numerical calculation of the dipole energy of a Néel wall and for the domain model calculations including an external OOP magnetic field.

[39] S. Tarasenko, A. Stankiewicz, V. Tarasenko, and J. Ferré, J. Magn. Magn. Mater. 189, 19 (1998).

[40] R. Ramchal, A. K. Schmid, M. Farle, and H. Poppa, Phys. Rev. B 69, 214401 (2004).

[41] M. Kronseder, J. Minár, J. Braun, S. Günther, G. Woltersdorf, H. Ebert, and C. H. Back, Phys. Rev. B 83, 132404 (2011).

[42] D. Sander, J. Phys.: Condens. Matter 16, R603 (2004).

[43] W. L. O’Brien, T. Droubay, and B. P. Tonner, Phys. Rev. B 54, 9297 (1996).

[44] H. Abe, K. Amemiya, D. Matsumura, S. Kitagawa, H. Watanabe, T. Yokoyama, and T. Ohta, J. Magn. Magn. Mater. 302, 86 (2006).

[45] M. Farle, Rep. Prog. Phys. 61, 755 (1998).

[46] W. Kuch, J. Gilles, S. S. Kang, S. Imada, S. Suga, and J. Kirschner, Phys. Rev. B 62, 3824 (2000).

[47] G. Lauhoff, J. A. C. Bland, J. Lee, S. Langridge, and J. Penfold, Phys. Rev. B 60, 4087 (1999).

[48] P. Talagala, P. S. Fodor, D. Haddad, R. Naik, L. E. Wenger, P. P. Vaishnava, and V. M. Naik, Phys. Rev. B 66, 144426 (2002).

[49] F. Wilhelm, P. Poulopoulos, G. Ceballos, H. Wende, K. Baberschke, P. Srivastava, D. Benea, H. Ebert, M. Angelakeris, N. K. Flevaris, D. Niarchos, A. Rogalev, and N. B. Brookes, Phys. Rev. Lett. 85, 413 (2000).

[50] J. Choi, J. Wu, C. Won, Y. Z. Wu, A. Scholl, A. Doran, T. Owens, and Z. Q. Qiu, Phys. Rev. Lett. 98, 207205 (2007).

[51] J. Wu, J. Choi, C. Won, Y. Z. Wu, A. Scholl, A. Doran, C. Hwang, and Z. Q. Qiu, Phys. Rev. B 79, 014429 (2009).

[52] N. Saratz, A. Lichtenberger, O. Portmann, U. Ramsperger, A. Vindigni, and D. Pescia, Phys. Rev. Lett. 104, 077203 (2010).

[53] N. Saratz, U. Ramsperger, A. Vindigni, and D. Pescia, Phys. Rev. B 82, 184416 (2010).

[54] M. Kronseder, T. N. G. Meier, M. Zimmermann, M. Buchner, M. Vogel, and C. H. Back, Nat. Commun. 6, 6832 (2015). 